\title{
CAPTURING USER BEHAVIOR IN E-LEARNING ENVIRONMENTS
}

\author{
Enric Mor, Julià Minguillón \\ Department of Computer Science, Multimedia and Telecommunication, Universitat Oberta de Catalunya, Barcelona, Spain \\ emor@uoc.edu,jminguillona@uoc.edu \\ Francesc Santanach \\ Learning Technologies, Universitat Oberta de Catalunya, Barcelona, Spain \\ fsantanach@uoc.edu
}

Keywords: $\quad$ user tracking, user behavior, web usage mining, e-learning environments

\begin{abstract}
In this paper we describe a proposal for embedding hidden marks in the web pages of a complex web site with the aim of simplifying the process of collecting and analyzing users' behavior. Usually, server log files are used for tracking user actions, but these files present typical drawbacks due to their huge size and the lack of discrimination between user actions and automated responses. We propose two different strategies for embedding usage marks into web server log files, action-oriented and content-oriented. Action marks are button-oriented and keep track of user behavior oriented to perform tasks thorough system buttons and menus. Content marks keep track of page loads and navigation thorough links pointing to contents that act as traps. Both strategies are complementary and can be used at once. A simple experiment designed to capture user behavior once the initial web site page has been loaded is described as a real example of such proposal usage, showing the advantages of reducing the number of relevant log entries and focusing on specific user actions.
\end{abstract}

\section{INTRODUCTION}

One of the most interesting possibilities in any elearning environment is tracking user navigational behavior for analysis purposes, as it may help to discover unusual facts about the system itself, users' behavior and also can provide useful information for adaptation and personalization purposes. For example, having a user navigational model (Kay and Lum, 2004) may be used to perform an automated usability evaluation (Hilbert and Redmiles, 2000), detecting whether the system web interface was properly designed or not, and where users find obstacles and difficulties to reach out their objectives (Marsico and Levialdi, 2004). This navigation model can be also used to improve the student learning experience; navigation behavior and learning indicators and can serve to tailor learning itineraries to specific student needs. In the case of a virtual learning environment, several data and information levels can be determined and thus, several analysis levels can be established, and different research questions can be answered with the aid of web mining tools. Instructional designers, teachers but also system designers need powerful tools for understanding all the information collected in a virtual learning environment in order to improve the learning process and, thus, user experience and satisfaction, by means of an adaptive (Paramythis and Loidl-Reisinger, 2004) environment: monitoring and interpreting the activities of its users (Thomas et al., 2003), inferring user requirements and preferences, and acting upon the available knowledge on its users. Therefore, the extraction of the real learning and training patterns can be of great utility, among other purposes, to determine the quality of the e-learning environment design and to evaluate the concordance degree among the usability requirements and the navigation behavior of the users, but especially for personalization purposes, tailoring the learning plans to the student background and needs (Mor and Minguillón, 2004). The information extracted from behavior and learning patterns must not be used in any way but for learning and teaching purposes. The main issue of user behavior analysis in large e-learning environments is the great amount of data available from different sources and the lack of specific data for analysis purposes. Usually, as users interact with the learning management system by means of a web browser, it is 
possible to track all interactions by examining the log files stored in the servers which support the learning management system. This is a well known technique known as web mining (Chi et al., 2000), but it suffers from several drawbacks when complex (in both size and internal structure) sites are evaluated. Instead of analyzing raw log files directly from the servers, we propose to establish a minimum set of marks in strategic areas which is considered to be relevant for analysis purposes, with the double aim of capturing users' real activity and intentions while reducing the volume of data needed to analyze them.

This paper is organized as follows: Section 2 describes the basic services and the interactions that are interesting to capture from a pedagogical point of view. In Section 3 we describe the proposed mark embedding strategy for capturing these interactions comparing two different kinds of mark techniques. Section 4 describes the proposed framework for data collecting and analysis, and some preliminary results following a three level decomposition. Finally, the most important conclusions of this paper and current and future research lines are summarized in Section 5.

\section{E-LEARNING ENVIRONMENTS}

In the last years, learning management systems (LMS) and learning content management systems (LCMS) have become a common tool for teachers and institutions willing to set up virtual learning environments. In fact, both LMS and LCMS pursue the same goal, but focusing on the learning process or the learning contents, respectively, and they can be combined altogether, as in the UOC proprietary e-learning environment, the virtual campus. We are interested in capturing users activity when interacting with the LMS, that is, when accessing to the available services.

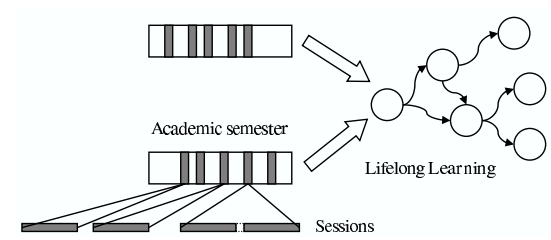

Figure 1: Hierarchical structure of navigation levels.

In the context of an e-learning environment, user activity and behavior can be analyzed from a threelevel (Mor et al., 2006) point of view, as shown in Figure 1. These three levels, namely short term, mid term and long term, are based on the study of student behavior during different time periods and, therefore, different data is collected at each level. The short term level, also known as session level, captures the way users navigate with particular goals in mind. It considers each student log into the LMS and the actions performed and the information accessed during the log session. At this level, as important what users do is how they do it, which pages they visit and how they reach them. The mid term level is the academic semester level and takes into account the single user sessions in a continuous flow during the course duration. At this level the main goal is to determine the navigational patterns followed by users but at a higher scale than in the previous level, according to some existing teaching plan, in the case of an e-learning environment. The third and long term level is called lifelong learning level and the main goal is to analyze how students evolve from the beginning of a degree until they successfully finish it.

Studying the user activity using this three level approach allows teachers, instructional designers and usability experts to obtain an accurate description and provides a complete view of system usage and user behavior, because at each level the user's goals can be learned and correlated with the data analysis results (Spiliopoulou, 2000). In this work we are focused on the collection, management and processing of web server logs and how can be used within the three-level framework in order to discover knowledge about user activity and behavior, specially for the first two levels.

\subsection{THE UOC virtual campus}

The Universitat Oberta de Catalunya (UOC ${ }^{1}$, in English known as Open University of Catalonia) is a completely online university which offers 19 official degrees, several graduate programs and post-graduate studies, and a doctoral degree, with almost 40000 students and more than 1800 people including instructional designers, teachers, tutors, academic and technical staff, and so. The UOC virtual campus is an integrated web based e-learning environment which allows users to asynchronously communicate with other users using a mail system, and includes an agenda, a news service, virtual classrooms, a digital library and other e-learning related tools (Sangrà, 2002). Each subject has a virtual classroom with all the elements and tools for the learning/teaching process.

\subsection{System architecture}

The UOC virtual campus is built upon a complex database server system which uses a hierarchical structure of servers which deal with different kinds

\footnotetext{
${ }^{1}$ http: //www . uoc.edu
} 
of user requests. There are up to 24 front-end servers (depending on the server load at each moment), and an automated load-balance system moves each user login to the front-end with lower load at that moment, switching on and off the total number of frontends depending on system load. At peak hours, it is not uncommon to achieve 3000 concurrent connections, while 1500 is the average number of concurrent users in office hours. Briefly, the virtual campus uses client-server web technology and common interface to integrate a series of services and functionalities. These functionalities include: access to online educational materials, library resources and general academic and cultural information; student management enquiries service; and interaction with professors and other students through pre-defined communication channels (e.g., forums, activity spaces). Services include: an email account; a collection of virtual classrooms, where each one has several communication spaces where students and teachers can interact and share learning resources; a digital library which integrates all the digital and non-digital contents into the virtual campus. When users navigate through these services, they leave a track which can be posteriorly analyzed for user modelling purposes. Most of this information is collected by the web servers in form of server log files, according to the Apache Common Log Format (CLF). Following the classical architecture of web information systems, there are three different layers for handling all the requests from the users of the web site. A first layer, the presentation layer, is handled by the web servers, which generate access logs with all the interactions (i.e. clicks) performed by users. Most of these interactions are not only navigational but also involve the use of some services and components, handled by the business logics layer, which does also generate its own program logs in different formats, according to each application. The data model layer is handled by a back-end database which stores all the data generated by the applications in the previous layer, which can also be traced for analysis purposes (i.e. who did the last change on a specific record). Figure 2 shows this three-level architecture.

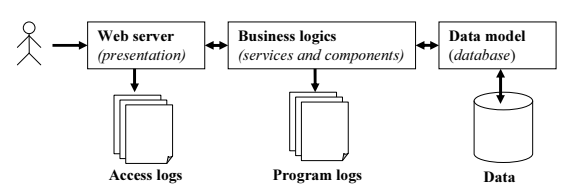

Figure 2: E-learning environment data architecture.

\subsection{Server $\log$ files}

For each action a user performs in the virtual campus, one or more lines representing such action are logged in several servers. Furthermore, depending on the type of action, several servers might log the same action but using different information. In this work we have used mainly the log files from the Apache servers which act as front-ends, once them have been joined in a single file which is generated each day. This file is firstly preprocessed in order to remove all those log lines which are surely not hits produced by the user, such as the load of icons, style sheets, banners, and so. This preprocessing reduces the amount of lines in a $90 \%$. Nevertheless, during a typical week, the total number of lines that needs to be processed is still about 36 million lines, approximately $12 \mathrm{~GB}$, which is indeed a very large figure. Therefore, a second preprocessing oriented towards narrowing the experiment is required, following the recommendations described in (Cooley et al., 1999).

Users can be uniquely identified because there is a unique session number generated each time a user logs into the virtual campus using his or her username and password. IP addresses are discarded because there is the possibility of many user accessing through the same proxy server which might mask the real IP address. Therefore, it is possible not only to identify individual users but also each individual session, which is useful to establish the different navigational levels described in the following section. Lines without session number are removed. Server log files are generated on-the-fly by the 24 web servers, and these files are copied onto other server which joins them and applies the filters aforementioned above, in a 24 hour basis routine, starting at 01:00am. A simple analysis of connection patterns reveals that the lowest server load is between 04:00am and 05:00am, so consecutive log files are joined and split again at such time. These daily (or weekly) chunks of preprocessed $\log$ files are the starting point for analysis purposes.

\section{MARKING STRATEGY}

Our goal is to capture the real intentions of each user navigating through the e-learning environment, but focusing on those activities related to the learning process (accessing the mailbox and virtual classrooms, using learning resources, and so). From the starting page, a set of marks is introduced in the most likely paths followed by the learners, in order to validate such paths and trying to discover any interesting fact that might be relevant. 


\subsection{Action based marking strategy}

Each click a learner performs in a critic (i.e. important to the learning process) element of a web page, a call to an application in the business logics layer is performed, and this generates one or more lines in the web server log files. The simplest way to capture such click is adding a special parameter indicating the action performed by the learner, according to the context where such element appears. Therefore, adding the string "\&ACTION=name" (where name identifies the action being performed by the user) to each relevant link causes such string to be logged and, therefore, easily traceable. For analysis purposes, it is important to choose action names that are not prefixes one of the other, simplifying the use of "grep" or similar tools for inspecting the generated log file. The main drawback of this strategy is that it might interfere in the correct functioning of the application programs handling each call. For example, if the handling servlet or CGI has a security mechanism for checking the received parameters, this mechanism should be modified in order to incorporate the new parameter. This may be very expensive for complex web sites where hundreds or even thousands of services available through servelts or CGI are already running.

\subsection{Content based marking strategy}

The second strategy tries to hide "transparent" resources in the web pages, which generate a specific entry in the log file when such pages are loaded (partially or completely, depending on the place where they are embedded). This is typically achieved by adding an invisible (transparent) tiny image in the web page, unnoticeable by the user but that will generate a new entry in the server log file when loaded, using a simple HTML sentence like the following:

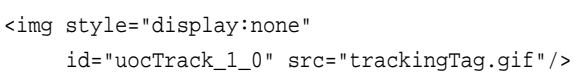

This is used to capture when a learner reaches a web page considered relevant for the learning process. This approach is also useful to generate a log entry when a link in a web page is accessed. This is done by forcing the invisible image to be loaded when the link is clicked, as shown in the following HTML sentences:

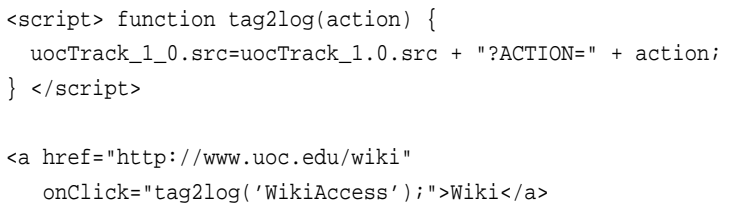

This strategy is more time consuming than the previous one, but it has a main advantage: it does not depend on any particular call, it does not interfere with any existing servlet or CGI, and it can be automated in order to be incorporated in all the web pages containing a particular resource or service that has been selected to be logged.

\subsection{Example}

Both marking strategies can be used at the same time, recording different kinds of user behavior. In complex web sites where the same goal can be reached from different paths, it is necessary to know not only user actions but also other places visited as well, in order to build a better user model for analysis purposes. The following fragment of a preprocessed log file shows marks from both types, action based and content based. Notice that this file is simpler than the equivalent Apache CLF one, as the IP address, timestamp and other fields have been replaced by user and session identifiers and so.

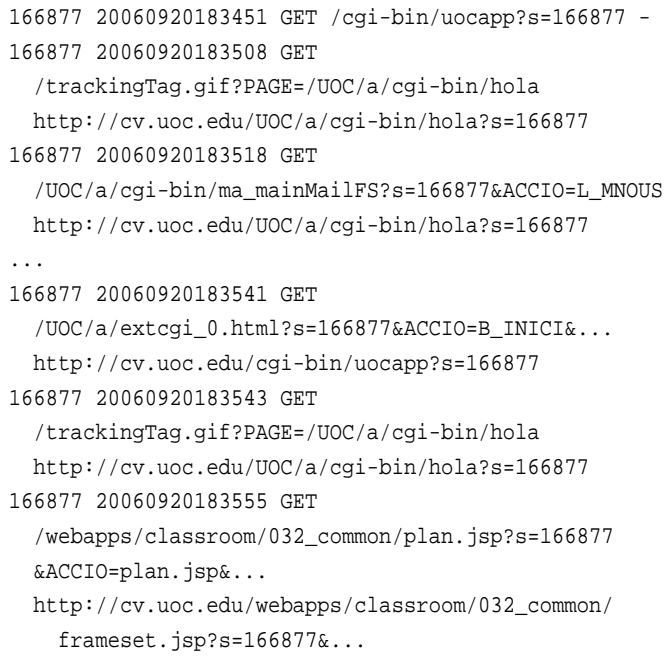

In this case, a student with identifier 166877 logs in into the virtual campus, it loads the initial web page is loaded (trackingTag.gif?PAGE), and he or she accesses the mailbox (ACCIO=L_MNOUS). Then, he or she goes back to the initial web page $\left(\mathrm{ACCIO}=\mathrm{B} \_I N I C I\right)$, which is loaded again, and finally goes to one of the virtual classrooms he or she is enrolled to $(\mathrm{ACCIO}=\mathrm{plan} \cdot \mathrm{jsp})$.

\subsection{Establishing a methodology}

One of the most important results of the embedding mark proposal is that provides the web administrators a simple but realistic map of the available services in the e-learning environment and how they are 
used by learners (or site users in general). Without this map, any effort to understand user behavior becomes too expensive due to the necessity of identifying the specific entries in log server files related to the activity being monitored. Even trying to reproduce previous experiments (Mor et al., 2006) for validating them with new available data becomes very difficult if the virtual environment has changed even only slightly, which is not uncommon in complex web environments. Therefore, any embedding mark process should be done according to the following requirements: (a) Architecture independent: all the changes in the web site should be done in the presentation layer only, and not in the business logics layer; (b) Scalability: the proposed embedding mark strategy should be the same independently of the size of the web site being monitored; (c) Consistency: all the relevant information needed for usage analysis is present in the log files; (d) Hierarchical: in order to broad or narrow each experiment carried out, trees and subtrees of marks should be activated and deactivated easily. For example, "analyze only actions related to the virtual classroom"; (e) Technology independent: any change in the technological aspects of the business logics layer should not interfere with the embedded marking system; and (f) Automatization: a complex web site with thousands of web pages should be able to be modified by means of an automated script.

Studying user behavior in a web site needs to establish which elements and actions will be monitored and further analyzed. In a web site with a wide range of services and goals available to users, it will be necessary to identify the main user tasks and the possible user actions related to such tasks as well. Using the cognitive walkthrough method (Wharton et al., 1994) it is possible to obtain a first version of the sequence of steps that users perform in order to achieve a specific task. Then, starting from this sequence and the knowledge of the services to be analyzed, a initial set of marks can be established for generating a first map of the web site. This map is analyzed to discover any gap due to an incomplete task description. This process is repeated until a reasonable map is obtained.

\section{EXPERIMENTAL RESULTS}

We provide a simple experiment showing user behavior once they just log in into the virtual campus. Our goal is to determine the paths followed by the students once they $\log$ in into the virtual campus, which is interesting because the same goal can be reached from different places, thus evaluating web design and the relative importance of the available elements.

\subsection{Data set}

The Fall 2006 academic semester started September the 20th, 2006. This is a special day because most users log in motivated by the fact that it is the first day, and they want to obtain information about the subjects they have enrolled into, the available resources, and so. The preprocessed log file for such day is 7,8 million lines long (around 1,5 GB), containing information about 105000 sessions, with almost 42000 student sessions from 20000 different students. We have selected 30000 sessions of a subset of 14600 students for this experiment, concretely those studying any official degree in Catalan. On the other hand, a simple inspection reveals that some sessions show a bizarre behavior, probably caused by a malfunctioning of the login process. For example, there are very short sessions with no interaction, which are removed, using a threshold of 3 seconds, the minimum amount of time for loading the initial page using a fast connection. From the initial web page, students have access to: (a) The mailbox, from three different buttons or links: a message notification with the amount of new mails, an icon representing a flag which means that there are new messages waiting, and a main mailbox access; (b) The list of subjects where the learner is enrolled into; (c) The virtual classroom for each one of the subjects the learner is enrolled into; (d) The notice boards and forums for each one of the subjects the learner is enrolled into; and (e) Other services available (digital library, community, profile, and so).

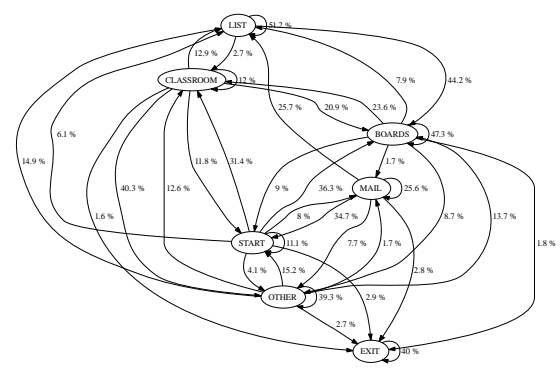

Figure 3: Most frequent navigational paths.

Results show that $49,5 \%$ of the students directly go to the notice boards and forums as their first action. This is normal in the first day of an academic semester, as students read the welcome messages and some of them write short messages introducing themselves as well. In second position, a $26,4 \%$ of the students go to one of the virtual classrooms. A $12,0 \%$ of the students go to the mailbox by means of the message notification link, while only a $1,7 \%$ and $0,6 \%$ access the mailbox through the main button and the flag icon respectively. A $8,1 \%$ of the students go to the 
list of subjects, and the remaining 1,7\% (which is a remarkably low figure) perform other actions. Figure 3 shows the most frequent paths followed by learners between the different spaces in the virtual campus. Only relevant links between nodes have been drawn. A link is relevant when the percentage of students that follow such link is at least a 5\% for both nodes. Notice that this graph includes all the possible paths, that is, not only the first action taken from the initial page.

\section{CONCLUSION}

In this paper a proposal for embedding hidden marks in web pages with the aim of user action identification has been proposed. Two complementary marking strategies have been described: the first one, action based, consists in adding special parameters in the calls to applications in the business logics layer of a web site, in order to capture the specific clicks in the relevant elements for analysis purposes. The second one, content based, consists in hiding invisible pieces of content that, combined with simple scripts, generate $\log$ entries for both user actions and navigation paths. Preliminary results show that this approach is useful to face both well known problems when analyzing large and complex web sites. First, users's intentions are better captured as only the relevant actions (for analysis purposes) are logged. Second, generated data are only a small fraction of the original data stored in the web server log files, thus reducing both time and space requirements for off-line processing. A simple experiment showing user behavior the first day of the academic semester has been described. This experiment reveals interesting information, for example, students start sessions reading all the welcome messages from the teacher and other students, and replying such messages. This fact could be used to design a specific initial page for the first session during first day, when all the other available services are not requested. Similar ideas for improving the e-learning environment can be extracted from the results of each activity analysis, depending on the educational context.

Current and future research in this topic should include the use of large scale web usage mining techniques for clustering purposes. Analyzing the evolution of users' behavior along a period of time is also an interesting issue. Finally, as web services are becoming more "semantic", it will be important to establish the appropriate relationships between the new services available in the virtual campus and real user intentions when searching or browsing such services.

\section{ACKNOWLEDGEMENTS}

This work has been partially supported by a Spanish government grant under the project PERSONAL (TIN2006-15107-C02-01).

\section{REFERENCES}

Chi, E. H., Pirolli, P., and Pitkow, J. (2000). The scent of a site: a system for analyzing and predicting information scent, usage, and usability of a web site. In Proceedings of the SIGCHI conference on Human factors in computing systems, pages 161-168, New York, NY, USA. ACM Press.

Cooley, R., Mobasher, B., and Srivastava, J. (1999). Data preparation for mining world wide web browsing patterns. Knowledge and Information Systems, 1(1):532.

Hilbert, D. M. and Redmiles, D. F. (2000). Extracting usability information from user interface events. ACM Computing Surveys, 32(4):384-421.

Kay, J. and Lum, A. (2004). Creating user models from web logs. In Proceedings of the Intelligent User Interfaces Workshop: Behavior-Based User Interface Customization.

Marsico, M. D. and Levialdi, S. (2004). Evaluating web sites: exploiting user's expectations. International Journal of Human-Computer Studies, 60(3):381-416.

Mor, E. and Minguillón, J. (2004). E-learning personalization based on itineraries and long-term navigational behavior. In Proceedings of the Thirteenth International World Wide Web Conference, volume 2, pages 264-265, New York, NY, USA. ACM Press.

Mor, E., Minguillón, J., and Carbó, J. M. (2006). Data Mining in E-learning, chapter Analysis of User Navigational Behavior for E-Learning Personalization, pages 227-246. WIT Press.

Paramythis, A. and Loidl-Reisinger, S. (2004). Adaptive learning environments and e-learning standards. Electronic Journal of e-Learning, 2(2).

Sangrà, A. (2002). A new learning model for the information and knowledge society: The case of the UOC. International Review of Research in Open and Distance Learning, 2(2).

Spiliopoulou, M. (2000). Web usage mining for web site evaluation. Communications of the ACM, 43(8):127134.

Thomas, R., Kennedy, G., Draper, S., Mancy, R., Crease, M., Evans, H., and Gray, P. (2003). Generic usage monitoring of programming students. In Proceedings of the ASCILITE 2003 Conference, pages 715-719, Adelaide, Australia.

Wharton, C., Rieman, J., Lewis, C., and Polson, P. (1994). Usability inspection methods, chapter The cognitive walkthrough method: a practitioner's guide, pages 105-140. John Wiley \& Sons, Inc. 